\title{
From a Categorical Point of View: K-nets as Limit Denotators
}

\author{
Guerino Mazzola, Universität Zürich, \\ Institut für Informatik \\ e-mail: guerino@mazzola.ch \\ Moreno Andreatta, IRCAM/CNRS \\ Equipe Représentations Musicales \\ e-mail: Moreno.Andreatta@ircam.fr
}

October 1, 2005

\begin{abstract}
We present the interpretation of Klumpenhouwer Networks as a special limit construction in the framework of the topos-theoretic denotator architecture developed in [19]. This approach at once offers (1) a vast generalization of the network concept, including networks of dodecaphonic series, for example, and (2) the formally closed recursive construction of iterated networks, i.e., networks of networks of networks, ..., without changing the construction principle.
\end{abstract}

\section{Introduction}

The shift from a set-theoretical to a transformational approach is one of the most intriguing and promising achievement of modern music theory. A study of the emergence of the group concept in music theory, analysis, and composition shows that the transformational perspective arises independently and almost contemporaneously in the American and European traditions in the theoretical writings of some mathematically-inclined theorists and composers, in particular Wolfgang Graeser, Milton Babbitt, Iannis Xenakis and Anatol Vieru [1]. They all developed theoretical models of the truly musical duality between "tones" and "intervals" by considering the notion of an algebraic structure as a basic theoretical concept for explaining this duality. In fact, after Graeser's group-theoretical study of symmetries in Bach's Art 
of Fugue, with Milton Babbitt's algebraic formalization of the Twelve-Tone System, Xenakis' sieve-theoretical constructions of musical (ordered) structures, and Vieru's group-theoretical description of modalism, music theory gradually shifts from the study of the properties of collections of elements to the study of the relations between these elements and, more crucially, to the operations upon them.

Modern algebra is the natural theoretical framework for the new transformational perspective since it enables a precise characterization of the concept of mathematical structure as a collection of elements together with (internal) relations and operations between them. The application of algebraic methods in Twentieth-Century music theory, analysis, and composition has therefore strong consequences in the evolution of transformation(al) theory as an autonomous discipline in the music-theoretical and analytical community. Such transformational methods were the basis of the categorical approach of one of the authors in his book with the programmatic title "Gruppen und Kategorien in der Musik" [16]. Already in these "early days" of applications of modern mathematics to music theory, a special process diagram type was considered, giving rise to the so-called circle chords (they were actually introduced by one of the authors in a university course in 1981 and published in [16]). A circle chord is a local composition ${ }^{1} K \subset \mathbb{Z}_{12}$ such that there is a not necessarily invertible affine map $g: \mathbb{Z}_{12} \rightarrow \mathbb{Z}_{12}$, the monoid $\langle g\rangle$ generated by $g$ describing $K$ as follows. We select a pitch class $x$ and then successively apply all powers $g^{i}$ to $x$. So we have the sequence $x, g(x), g^{2}(x), \ldots g^{i}(x) \ldots$ Since the different entries in such a sequence are finite in number, we must have $g^{k+t}(x)=g^{k}(x)$ for determined minimal $k, t$. This means that we have a diagram scheme $e^{2} \Delta$

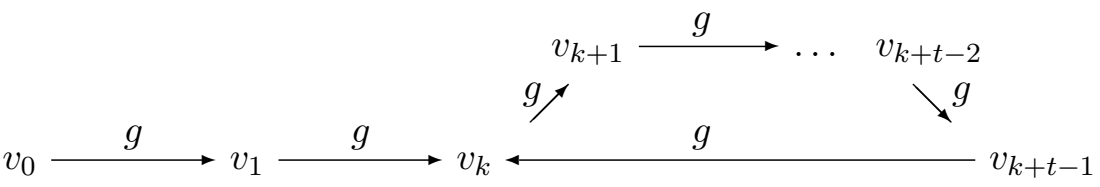

\footnotetext{
${ }^{1} \mathrm{~A}$ local composition is a pair $(K, M)$, where $K$ is a subset of a module $M$. In the special case of the cyclic group $M=\mathbb{Z}_{12}$, a local composition is simply a pitch class set $K \subset \mathbb{Z}_{12}$. This concept is a first approach to more complex musical structures, which can be described in terms of global compositions, i.e., the covering of a given score by a number of local compositions, see "Geometrie der Töne" [17] for details. For a critical account of this approach within the American tradition of music theory, see John Roeder's review of "Geometrie der Töne" in a previous issue of this journal [25].

${ }^{2}$ In the theory of diagrams, a diagram scheme is a digraph or quiver, i.e., a directed multigraph, possibly with loops. We also prefer to use the classical mathematical term "vertex" instead of "node", which is more established in music theory and computer science. For a modern presentation of graph theory and categories, we refer to [21].
} 
terminating with a backwards arrow from vertex $v_{k+t-1}$ to vertex $v_{k}$. The diagram $\mathcal{D}$ then associates the map $g$ with each arrow, and we have the "network", i.e., an element of $\lim \mathcal{D}$ consisting of the given sequence

$$
s=\left(x, g(x), g^{2}(x), \ldots g^{k}(x) \ldots g^{k+t-1}(x)\right) .
$$

The circle chords have been classified in [16] and yield only a small number of chords (isomorphism classes of pitch class sets), which are very common in classical harmony, among others the major and minor triad, see figure 1.

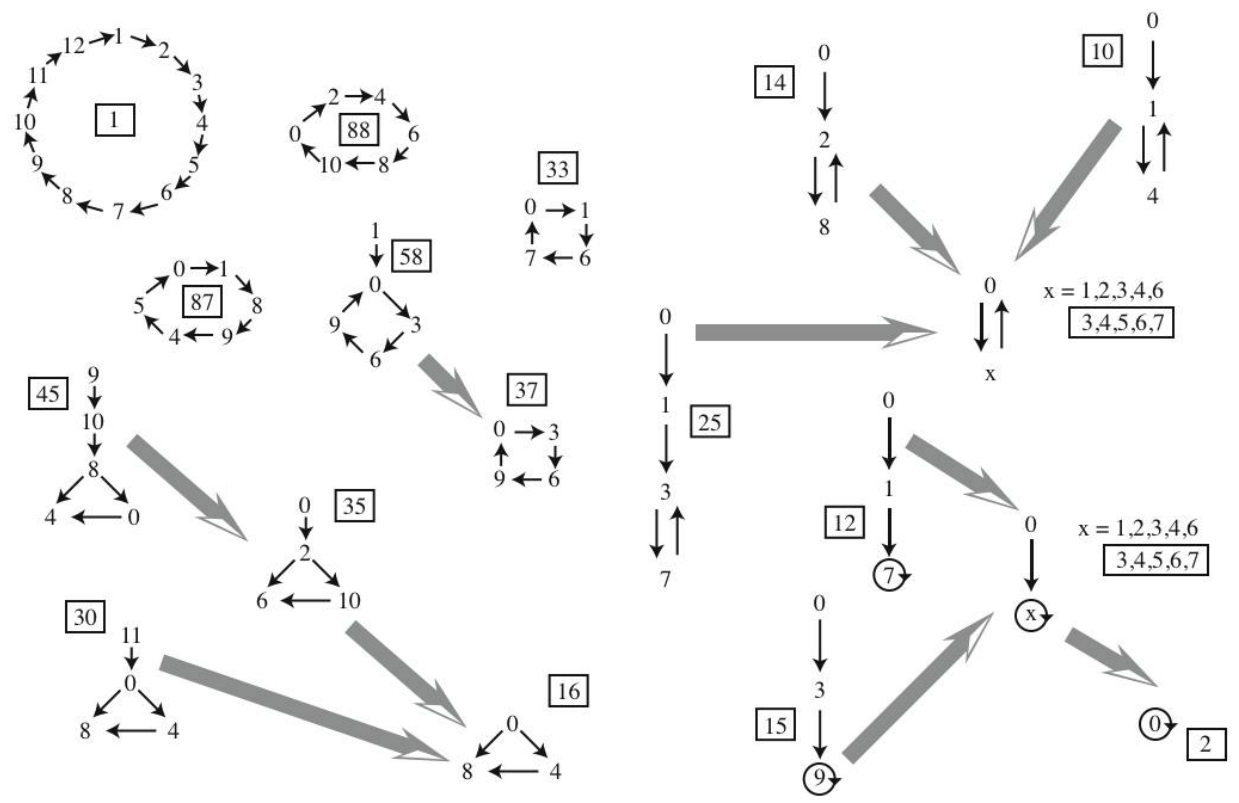

Figure 1: The 16 circle chords in $\mathbb{Z}_{12}$, together with their generating symmetry action (arrows). The numbers refer to the classification of chords in [19]. A large arrow from one chord to another means that the target chord is isomorphic to a subchord of the start chord, and that the subchord is generated by a suborbit of the same symmetry action as for the superchord.

This type of transformational method is also well represented by David Lewin's algebraic formalizations of traditional set-theoretical concepts that lead to some constructions, such as the Generalized Interval System. These formalizations are progressively shifting the analytical perspective from the description of abstract collection of elements and their relations to the construction of conceptual spaces, where the properties of elements are described by the transformation groups acting on them. 
Algebra therefore offers a natural explanation of the evolution of transformational techniques, in particular the concept of transformational graphs and networks $[10,11]$, which are now considered as a new theoretical paradigm in music analysis [3]. Going a step further than classical algebra, the evolution of abstract methods in Twentieth-Century mathematics suggests new paradigms for the formalization of musical structures. The most powerful mathematical framework dealing with the duality of objects and transformations is provided by category theory as it was introduced in the forties by Samuel Eilenberg and Saunders Mac Lane [6]. This approach has been applied to music by one of the authors as a general way of dealing with algebraic structures in music and it is now accepted as a common musictheoretical paradigm in European music theory. It is curious to notice that in the American set-theoretical tradition, categorical concepts have been widely ignored, even by theorists, such David Lewin, whose theoretical construction naturally have a categorical flavour.

There is however a noteworthy singular example in the American tradition which explicitly mentions a possible transfer of some concepts of category theory to music theory. In an paper discussing some measures of Arnold Schoenberg's Trio Op. 45 [23], John Peel already in 1975 proposed the use of commutative diagrams in order to turn the space of tetrachords into a category suitable for covariant functors and other tools of category theory. Unfortunately, category theory remains almost completely confined to the ample footnotes, and the categorical constructions seem to obscure Schoenberg's celebrated measures instead of clarifying their musical properties. However, the a priori use of diagrams in the sense of Klumpenhouwer networks (or K-nets) and even supernets (!) is unambiguously traced in this visionary paper. The time for a well-motivated application of category theory to music still had to come.

It turns out that some recent developments of transformational methods, leading in particular to the K-nets, have a natural connection with the categorical concept framework of denotators as described in the comprehensive study The Topos of Music [20]. The genesis and the place of $\mathrm{K}$-nets in contemporary music theory is well documented by Lewin $[12,14]$ and Klumpenhouwer [9] and many music-theorists, analysts, and composers who discussed the potentialities of this approach in the study of particular Twelve-Tone constructions $[4,5,15,24]$. In a more philosophical perspective, the shift to networks and categories expresses a change of the understanding of the musical dynamics to a diagrammatic language. But this is more than a technical procedure, since the category of diagrams has a rich logical structure, which is well described by topos theory. We will briefly give an 
idea of the topos-theoretical description of transformational theory in our presentation of K-nets by usage of the category directed graphs.

Here we would like to stress a general problem naturally arising when dealing with such abstract structures without using the powerful tools of category theory. Klumpenhouwer networks and, more generally, David Lewin's transformational graphs and networks, are considered as configurations of nodes and arrows, together with transformations labeling these arrows. According to Klumpenhower [8], such a theoretical framework "can be applied to generate multiple levels of structure and to emphasize recursive relations between various levels". The problem of such a construction is that higher level networks would require the introduction of higher level node objects. This is only possibly by use of so-called limits as they are provided by category theory. By recursively generalizing the object/operation duality at any level of description, category theory enables the music theorist to precisely deal with these two fundamental concepts. At the same time it suggests how one can use the same theoretical framework to take into account musical properties which one can no longer analyze in the traditional $T / I$-group context provided by K-nets constructions.

\section{Preliminary Remarks on Automorphism Groups and Digraphs}

This section serves a double purpose: First it introduces some basic notations about groups and morphisms involved in network theory, and second, it reviews some results concerning related automorphism groups as originally studied in the K-net context in [12].

The original theory is modeled on pitch classes (pc), the set of pitch classes is the cyclic group $\mathbb{Z}_{12}$ of cosets $\bar{x}=x+12 \cdot \mathbb{Z}$ of integers $x \bmod 12$. If no confusion is likely, we also use the integers $x$ representing $\bar{x}$ to denote pitch classes. We also use the structure of a commutative ring on $\mathbb{Z}_{12}$, which is given by the product $\bar{x} \cdot \bar{y}=\overline{x \cdot y}$ induced by the multiplication of integers. In this ring, there are four invertible elements, viz. $x=1,5,7,11$, each of them being its own inverse, we denote their set by $\mathbb{Z}^{*}=\{1,5,7,11\}$. The full group $G A\left(\mathbb{Z}_{12}\right)$ of affine automorphisms of $\mathbb{Z}_{12}$ is by definitiion the group of bijections $e^{t} p: \mathbb{Z}_{12} \rightarrow \mathbb{Z}_{12}$ defined by $e^{t} p(x)=t+p \cdot x$, where $p \in \mathbb{Z}^{*}$. The composition of such automorphisms $e^{t} p, e^{s} q \in G A\left(\mathbb{Z}_{12}\right)$ is

$$
e^{t} p \circ e^{s} q=e^{t+p s} p q,
$$


whereas the inverse of $e^{t} p$ is

$$
\left(e^{t} p\right)^{-1}=e^{-p t} p
$$

The group $G A\left(\mathbb{Z}_{12}\right)$ is a semi-direct product of its normal subgroup $e^{\mathbb{Z}_{12}}=$ $\left\{e^{t} 1 \mid t \in \mathbb{Z}_{12}\right\}$ of translations (i.e., transpositions in the pitch class context) and the subgroup $e^{0} \mathbb{Z}_{12}^{*}=\left\{e^{0} p \mid p \in \mathbb{Z}^{*}\right\}$ of linear automorphisms, i.e., the identity $e^{0} 1$, the inversion (in zero) $e^{0} 11$, the fourth dilatation $e^{0} 5$, and the fifth dilatation $e^{0} 7$. We also write these operations by omission of the trivial translation parts, i.e., 11 instead of $e^{0} 11$. The semidirect product structure means that we have a cartesian product decomposition $G A\left(\mathbb{Z}_{12}\right) \stackrel{\sim}{\rightarrow} \mathbb{Z}_{12} \rtimes \mathbb{Z}_{12}^{*}: e^{t} p \mapsto(t, p)$, and that the group product $(t, p) \cdot(s, q)=(t+p s, p q)$ to the right is induced by the action $\mathbb{Z}_{12}^{*} \rightarrow \operatorname{Aut}\left(\mathbb{Z}_{12}\right)$ of multiplication by an invertible element. In network theory, one considers the subgroup of those automorphisms which are generated by inversions and translations. Their group is called $T / I$ group, we denote it by $T / I$. We therefore have a subgroup $T / I \subset G A\left(\mathbb{Z}_{12}\right)$, which is identified with the semidirect product subgroup $\mathbb{Z}_{12} \rtimes\{ \pm 1\} \subset \mathbb{Z}_{12} \rtimes \mathbb{Z}_{12}^{*}$ of the translation group and the two-element group $\{ \pm 1\}$ generated by the zero inversion $11=-1$. The automorphism group of $T / I$ is easily calculated. In fact, the cyclic subgroup $\mathbb{Z}_{12}$ of translations is generated by the unit translation $e^{1}$ of order 12. Hence its image under an automorphism $f: T / I \stackrel{\sim}{\rightarrow} T / I$ is also of order 12. If $f\left(e^{1}\right)=e^{t} 11$, then this element would evidently have order two. Therefore $f\left(e^{1}\right)=e^{t}$, in other words, translations are mapped bijectively onto translations. Therefore, on $\mathbb{Z}_{12}, f$ is just a group automorphism, i.e., the multiplication by an invertible element $p \in \mathbb{Z}_{12}^{*}$. On the other hand, we must have $f(11)=e^{s} 11$, since the bijection $f$ cannot map the inversion 11 to an element of the translation group. Therefore an automorphism $f$ of $T / I$ is determined by the invertible $p$ which describes $f$ on the translations, and the value $f(11)=e^{s} 11$ on the zero inversion. It is easy to verify that any such pair of data $(s, p) \in \mathbb{Z}_{12} \times \mathbb{Z}_{12}^{*}$ determines an automorphism $\langle s, p\rangle: T / I \stackrel{\sim}{\rightarrow} T / I$ with $\langle s, p\rangle\left(e^{t}\right)=e^{p t},\langle s, p\rangle(11)=e^{s} 11$. Further, the composition $\langle r, q\rangle \circ\langle s, p\rangle$ of two such automorphisms is

$$
\langle r, q\rangle \circ\langle s, p\rangle=\langle r+q s, q p\rangle,
$$

which means that we have an isomorphism of semi-direct product groups

$$
\operatorname{Aut}(T / I) \stackrel{\sim}{\rightarrow} \mathbb{Z}_{12} \rtimes \mathbb{Z}_{12}^{*} \stackrel{\sim}{\rightarrow} G A\left(\mathbb{Z}_{12}\right):\langle s, p\rangle \mapsto e^{s} p .
$$

To terminate these preliminary remarks, observe that we have the usual conjugation Int $: G A\left(\mathbb{Z}_{12}\right) \rightarrow \operatorname{Aut}(T / I)$, which sends $x \in G A\left(\mathbb{Z}_{12}\right)$ to the 
inner or conjugation automorphism $\operatorname{Int}(x)(y)=x \circ y \circ x^{-1}$ on $T / I$. Observe in particular that conjugation with any such $x$, not only elements of $T / I$, leaves $T / I$ invariant. If $x=e^{s} p$, then we have $\operatorname{Int}\left(e^{s} p\right)=\langle 2 s, p\rangle$. I.e., the inner automorphisms are a group isomorphic to the subgroup $2 \mathbb{Z}_{12} \rtimes \mathbb{Z}_{12}^{*}$ of index 2 in $A u t(T / I)$. In other words, an automorphism $\langle s, p\rangle$ is inner iff $s$ is even (and no further condition is imposed on $p$ ).

In the sequel, we shall view $T / I$ as being a category as follows: It has just one object, i.e., $\mathbb{Z}_{12}$, and the automorphisms $f: \mathbb{Z}_{12} \stackrel{\sim}{\rightarrow} \mathbb{Z}_{12}$ from $T / I$, together with their usual composition, are the morphisms. Then an automorphism of the category $T / I$ is identified with a group automorphism in $\operatorname{Aut}(T / I)$. Observe that this category will be viewed as a subcategory of the category $\operatorname{Mod}_{\mathbb{Z}}$ of modules over the ring $\mathbb{Z}$ of integers. We shall come back to this category in section 3 .

We shall consider digraphs (short form of directed graphs), which are defined as follows. Given a finite set $V$ of vertexes and a finite set $A$ of arrows, the associated digraph is a set map $\Delta: A \rightarrow V^{2}$, which to every arrow $a \in A$ associates an ordered pair $(x, y)$ or vertexes, in signs $x \stackrel{a}{\longrightarrow} y$, where $x=\operatorname{tail}(a)$ is called the arrow's tail and $y=\operatorname{head}(a)$ is called the arrow's head. We explicitely include multiple arrows between the same tail and head, as well as loops, i.e., arrows whose heads and tails coincide, see figure 2 for an example.

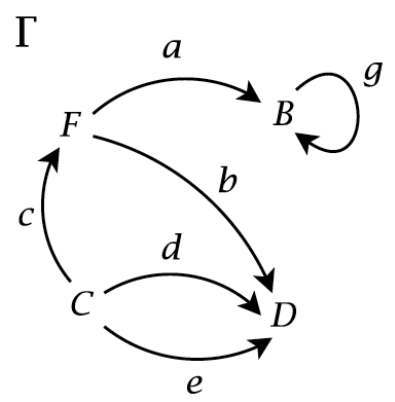

Figure 2: A digraph $\Gamma$ with vertexes $B, C, D, F$ and arrows $a, b, c, d, e, g$.

A path in a digraph $\Delta$ is a sequence $p=a_{1} a_{2} \ldots a_{l}$ of arrows of $\Delta$ such that the head of $a_{i}$ is identical to the tail of $a_{i-1}$ for all $i=1, \ldots l-1$. The integer $l$ is called the length of $p$ and is denoted by $l(p)$. Paths of length 0 are by definition single vertexes ("lazy paths"). Two paths $p=a_{1} a_{2} \ldots a_{l}, q=$ $b_{1} b_{2} \ldots b_{k}$ can be composed to the path $p q=a_{1} a_{2} \ldots a_{l} b_{1} b_{2} \ldots b_{k}$ if $\operatorname{tail}\left(a_{l}\right)=$ head $\left(b_{1}\right)$. With this composition rule, the set of paths defines the path category Path $(\Delta)$ of $\Delta$. 


\section{Reviewing the Original Approach by Lewin and Klumpenhouwer}

Referring to Lewin's original text [12], "Any network that uses $T$ and/or $I$ operations to interpret interrelations among pcs will be called a Klumpenhouwer Network." To be precise, the operations are the elements of the group $T / I$. And a Klumpenhouwer network (a K-net) is just a digraph, connecting pitch classes, which are placed on the digraph's vertexes, while the operations realize the digraph's arrows. This means that the operations connecting two vertexes transform the vertex pitch classes into each other. ${ }^{3}$ See figure 3 for an two examples of K-nets from Lewin's [12] and Klumpenhouwer's own work [8].
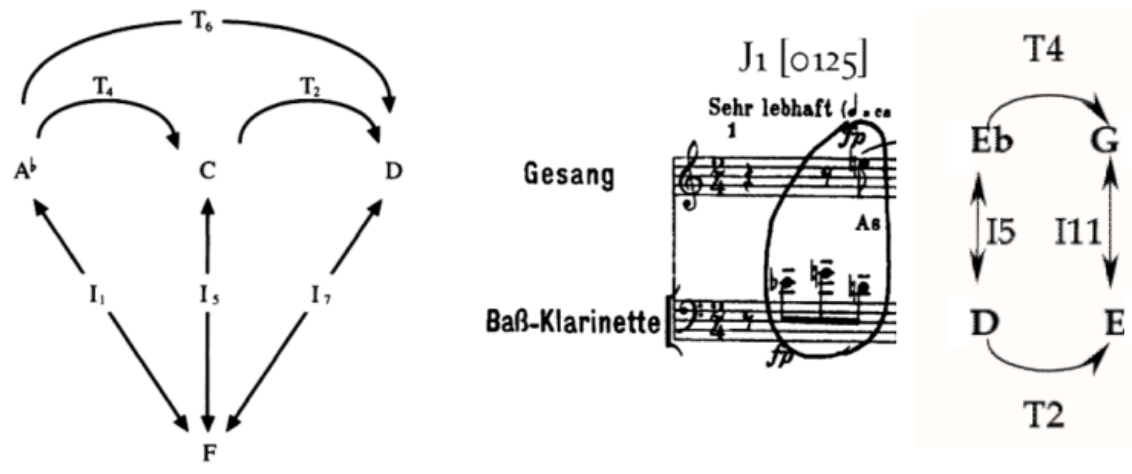

Figure 3: Two K-nets involving different digraphs. To the left, an example from Lewin's work [12]. To the right an example from Klumpenhouwer's unpublished work [8] on Webern's opus 16/4; it shows four vertexes in a pitch class set derived from the four-note set $\{\mathrm{Eb}, \mathrm{G}, \mathrm{D}, \mathrm{E}\}$ in the score shown in the middle.

So the network has two ingredients: the digraph with its arrows being interpreted by $T / I$-elements, and the pitch classes being placed in the vertex positions, together with the condition that under the given transformations, the pitch classes are transformed into each other. This configuration is an example of the well-known limit construction in classical mathematical category theory [6]. More precisely, to define a limit (of sets and set maps

\footnotetext{
${ }^{3}$ As Lewin remarked, the entire K-net theory could be generalized by including the $M$ operators, which are in fact affine operators. Robert Morris implicitely includes these operators in his definition of K-nets [22].
} 
in our case), one is given a digraph $\Delta: A \rightarrow V^{2}$ and a map $\mathcal{D}$ which for every vertex $v \in V$ defines a set $\mathcal{D}(v)$, and which for every arrow $x \stackrel{a}{\longrightarrow} y$ defines a map $\mathcal{D}(a): \mathcal{D}(x) \rightarrow \mathcal{D}(y)$. The map $\mathcal{D}$ is usually called a diagram (of sets and maps in our case), whereas $\Delta$ is called the underlying diagram scheme. The diagram canonically extends to a functor on the path category $\operatorname{Path}(\Delta)$ as follows. If $p=a_{1} a_{2} \ldots a_{l}$ is a path, then one sets $\mathcal{D}(p)=$ $\mathcal{D}\left(a_{1}\right) \circ \mathcal{D}\left(a_{2}\right) \circ \cdots \mathcal{D}\left(a_{l}\right)$.

The limit of $\mathcal{D}$ involves the following set. Within the cartesian product $\prod_{v \in V} \mathcal{D}(v)$, we select those tuples $t .=\left(t_{v}\right)_{v \in V}$ such that for every arrow $x \stackrel{a}{\longrightarrow} y$, we have $t_{y}=\mathcal{D}(a)\left(t_{x}\right)$. This set is denoted by $\lim \mathcal{D}$. Clearly, we have a projection map $p_{v}: \lim \mathcal{D} \rightarrow \mathcal{D}(v)$ for each vertex $v \in V$, and for every arrow $x \stackrel{a}{\longrightarrow} y$, we have the commutation condition $p_{y}=\mathcal{D}(a) \circ p_{x}$. To this generic construction, one may add so-called commutativity conditions. This means that for a number of pairs of paths $p, q: x \rightarrow y$ in $D$, one requires that $\mathcal{D}(p)=\mathcal{D}(q)$. If this is required for all pairs of paths starting and ending at the same vertex, the diagram is called a commutative diagram.

Clearly a Klumpenhouwer network is just one element $t . \in \lim \mathcal{D}$, where:

- the diagram scheme $\Delta$ is the network's digraph,

- the $T / I$ operations are the diagram maps between copies of the pitch classe set $\mathbb{Z}_{12}$, and

- the sequence of vertex entries defines one single element of $\lim \mathcal{D}$ (which in our case is a subset of the cartesian product of copies of $\mathbb{Z}_{12}$ ).

Note that in Klumpenhouwer's theory, it is required that the diagrams are commutative. But the general case doesn't need this condition. Two elements $t$., s. of one and the same $\operatorname{limit} \lim \mathcal{D}$ are what Lewin called strongly isographic in [12]. Clearly, strong isographies have no implications on the pitch class sets associated with networks. More precisely, if $t . \in \lim \mathcal{D}$, we derive the associated pitch class set $|t|=.\left\{t_{v} \mid v \in V\right\} \subset \mathbb{Z}_{12}$. Then, in general, the associated sets $|t|,. \mid s$. $\mid$ of isographic networks $t$., $s$. have no significant relation as sets. In particular they need not pertain to the same orbit under the canonical action of $T / I$. They need not even share their cardinality. Conversely, there are many different diagrams $\mathcal{D}_{1}, \mathcal{D}_{2}, \ldots$ with nets $t^{1} ., t^{2} ., \ldots$, respectively, giving rise to one and the same pitch class set $X=\left|t^{1}.\right|=\left|t^{2}.\right|=\ldots$. In other words, networks are the result of a strongly interpretative activity, much as a global composition is a result of a strong interpretative activity (see [19] for more details). 
From a more systematic point of view, networks and global compositions are not independent procedures. From the examples given by Klumpenhouwer and Lewin, one concludes that networks are not the first interpretative structures superimposed on a given composition. In the first preliminary instance, a composition $X$ is interpreted as a union of local "charts" $X=\bigcup_{i} X_{i}$, and then, after a projection of each chart $X_{i}$ onto its image $\bar{X}_{i} \subset \mathbb{Z}_{12}$ in the pitch class group, each such pitch class set is interpreted as the set $\bar{X}_{i}=\mid t^{i}$. $\mid$ associated with a network $t^{i}$. for an appropriate diagram $\mathcal{D}_{i}$. In other words, we are dealing with atlases of networks instead of atlases of local compositions as considered in the theory of global compositions, see [19, chapter 13]. Such atlases of networks are superimposed on given atlases of local compositions. This points towards a global theory of limit objects, but let us first study more closely the local approach in this paper.

In category theory, limits are related by so-called natural transformations. One very special case of such relations is addressed by Klumpenhouwer's concept of an "isomorphism" between networks. A more general setup introduces "isographies" and is described by Lewin [12]. We first discuss this approach and then come back to network isomorphisms. In Lewin's approach, one is given two diagrams $\mathcal{D}_{1}, \mathcal{D}_{2}$ on one and the same diagram scheme $\Delta$. An isography from $\mathcal{D}_{1}$ to $\mathcal{D}_{2}$ is an automorphism $f \in A u t(T / I)$ such that for any arrow $x \stackrel{a}{\longrightarrow} y$ in $\Delta$, we have $\mathcal{D}_{2}(a)=f\left(\mathcal{D}_{1}(a)\right)$. It is denoted by $f: \mathcal{D}_{1} \approx \mathcal{D}_{2}$.

Observe that isographies need not even have any networks in common, as is shown by the following example. Take the diagram scheme $\Delta$ consisting of one vertex $v$ and one loop $z$ in $v$. For the first diagram $\mathcal{D}_{1}$, we take the map $\mathcal{D}_{1}(z)=e^{2 q+1}(-1)$, for the second, we take $\mathcal{D}_{2}(z)=e^{2(2 q+1)}(-1)$. Then the first limit is empty, since this requires an impossible fixed point $x=(2 q+1)-x$. Therefore no network is possible here. The second limit is the set of those pitch classes $x$ such that $x=2(2 q+1)-x$, which is non-empty, so we have networks in this case. But one easily calculates that $\mathcal{D}_{2}(z)=\langle 2 q+1,1\rangle\left(\mathcal{D}_{1}(z)\right)$, hence the two diagrams are isographic.

There is however one special case of isographies, where one can tell more: If we require that the isography isomorphism $f: \mathcal{D}_{1} \approx \mathcal{D}_{2}$ is inner, i.e., $f=\langle 2 s, p\rangle$, then we have conjugation, i.e., there is $g \in T / I$ such that $\mathcal{D}_{2}(a)=f\left(\mathcal{D}_{1}(a)\right)=g \circ \mathcal{D}_{1}(a) g^{-1}$. This can be reread as the commutativity 
of the diagram

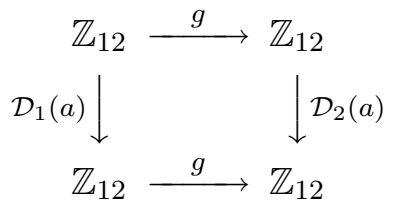

in the category $T / I$, for each arrow $a$ in $\Delta$. This is a special item of what in category theory is called a natural isomorphism between diagram $\mathcal{D}_{1}$ and diagram $\mathcal{D}_{2}$. In this case, it is easily seen that we also have an isomorphism $\lim \mathcal{D}_{1} \stackrel{\sim}{\rightarrow} \lim \mathcal{D}_{1}$ of limits, and therefore, the networks (i.e., elements) of $\mathcal{D}_{1}$ correspond one-to-one to networks (i.e., elements) of $\mathcal{D}_{2}$. More precisely, this correspondence is induced by the operation $g$ by sending a network $t .=\left(t_{v}\right)_{v \in V}$ to the network $g(t)=.\left(g\left(t_{v}\right)\right)_{v \in V}$. Therefore, networks which correspond under a network isomorphism induce associated sets $|t|,.|g(t)$. whch are isomorphic under $g$, i.e., $|g(t)|=.g|t$.$| .$

Summarizing: isomorphic networks are a lifting of associated isomorphic sets of pitch classes, while strongly isographic networks have only their defining diagram, but nothing else in common, and, finally, isographic diagrams have no implication on their networks, in general. The natural construction really is the network isomorphism, which is a special case of a natural transformation in category theory.

We should add that the above isomorphism concept is still a bit too special, also with regard to the proposed correspondencies between networks. In fact, Klumpenhouwer and Lewin describe networks of K-nets including operations which relate them, see figure 4 . The operations are not all the same (as $g$ from above), but may vary from vertex to vertex. Notice that all diagrams here are commutative.

So it is proposed that two diagrams $\mathcal{D}_{1}, \mathcal{D}_{2}$ over the same diagram scheme $\Delta$ are not necessarily related by a single operation (the $g$ above), but for every vertex $v \in V$ of the diagram scheme $\Delta$ one is given a specific operation $f(v): \mathcal{D}_{1}(v) \rightarrow \mathcal{D}_{2}(v)$ in $T / I$ such that the diagram

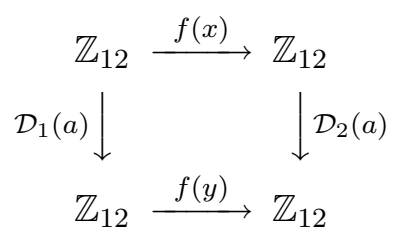

commutes for every arrow $x \stackrel{a}{\longrightarrow} y$ in $\Delta$ (recall that in our context we have $\mathcal{D}_{1}(v)=\mathbb{Z}_{12}$ for all vertexes $v$ ). Again, if this is the case, we have an isomorphism $f: \lim \mathcal{D}_{1} \stackrel{\sim}{\rightarrow} \lim \mathcal{D}_{2}$ of limits, taking the network $t$. to $f(t$.), 

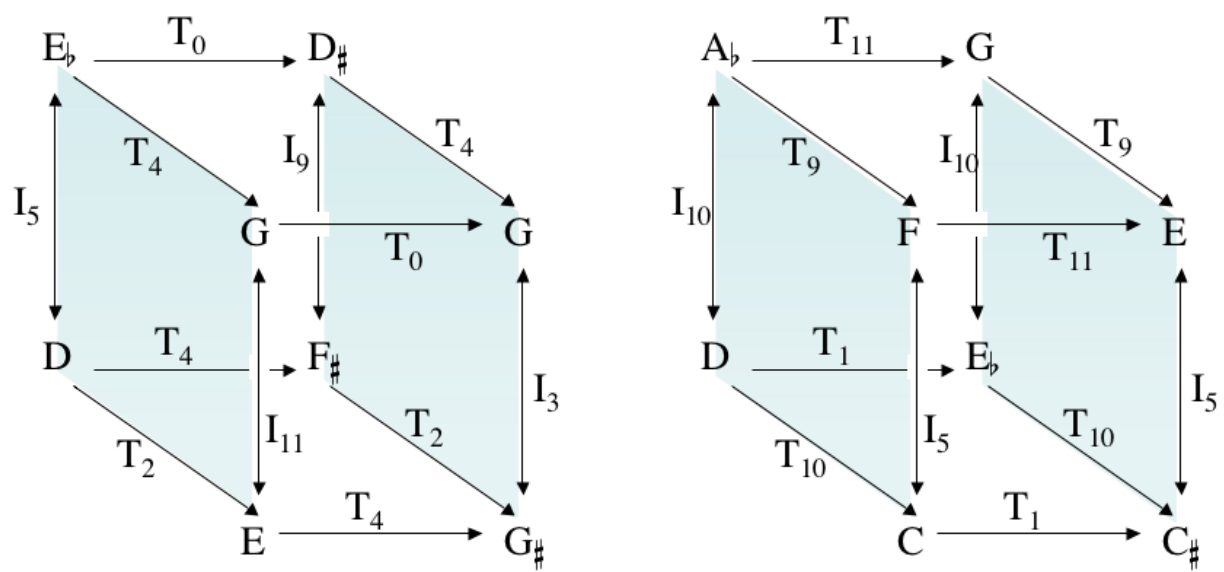

Figure 4: Two "networks" of K-nets used by Klumpenhouwer (left) [8] and Lewin (right) [12]. In each graphic, the 3D representation shows two Knets with shaded parallelogram shape, which are related by two pitch class transpositions, one for the upper vertex pairs, one for the lower pairs. The left K-nets are isographic, while the right ones are strongly isographic.

whose component in vertex $v$ is $f(v)\left(t_{v}\right)$. But observe that in this context, it is no longer true that networks $t ., f(t$.$) are necessarily associated with$ isomorphic sets $|t|,.|f(t)$.$| . In this setup, we are now looking at "networks$ of networks", and the question arises, which could be the general recursive procedure to step from a given iteration to the next higher one. Clearly, this requires a more systematic account on the nature of such networks. This is what we shall deal with in the next section.

But there is a minor generalization which we have to cope with, and which was discussed by Lewin in his analysis of Stockhausen's Klavierstück III [12]. Lewin's analysis deals not only with networks of single pitch classes, but of sets of pitch classes, see figure 5 .

The construction principle of such extended networks is immediate: The diagram $\mathcal{D}$, which yields sets $\mathcal{D}(v)$ and maps $\mathcal{D}(a): \mathcal{D}(x) \rightarrow \mathcal{D}(y)$, induces a second diagram $2^{\mathcal{D}}$, which is built on the same diagram scheme, but which, by definition, instead of $\mathcal{D}(v)$ produces the powerset $2^{4}(v)=2^{\mathcal{D}(v)}$, whereas for the arrow $x \stackrel{a}{\longrightarrow} y$, we have the powerset map $2^{\mathcal{D}}(a): 2^{\mathcal{D}}(x) \rightarrow 2^{\mathcal{D}}(y)$, where $2^{\mathcal{D}}(a)(X)=\{\mathcal{D}(a)(t) \mid t \in X\}$. This powerset diagram includes the previous one if one reinterprets elements $x \in \mathcal{D}(v)$ as singletons $\{x\} \in 2^{\mathcal{D}}(v)$.

\footnotetext{
${ }^{4}$ The powerset $2^{X}$ of a set $X$ is by definition the set of all subsets of $X$.
} 


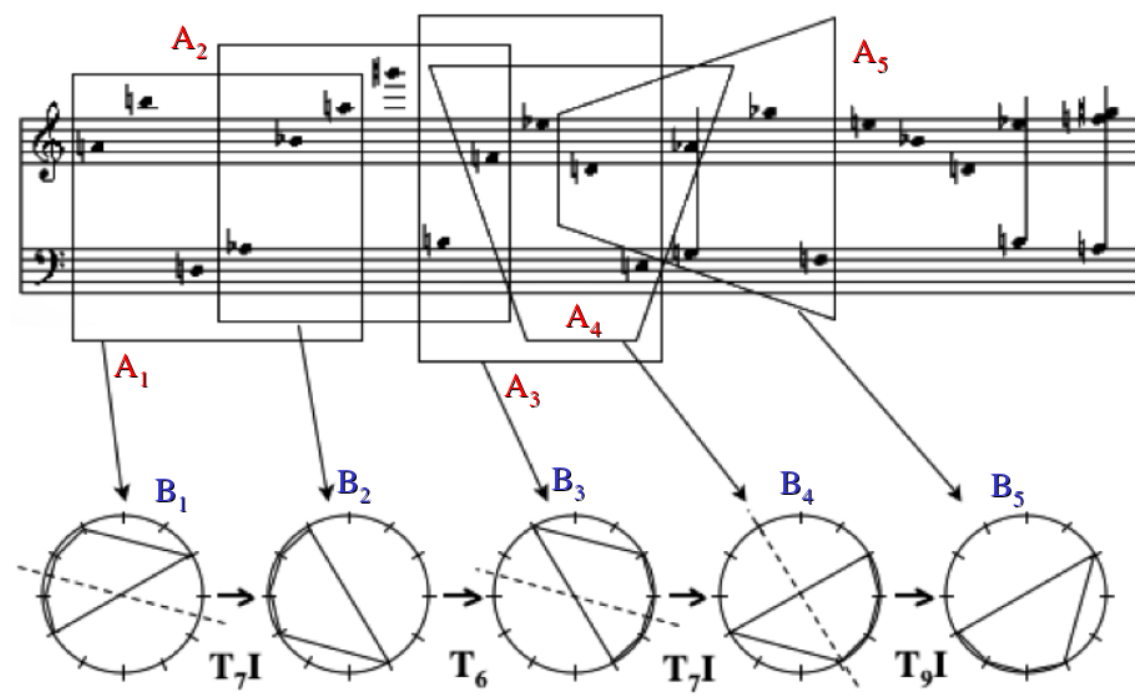

Figure 5: A covering of part of Stockhausen's Klavierstück III by charts $\mathrm{A}_{1}, \mathrm{~A}_{2}, \ldots$, which project to sets $\mathrm{B}_{1}, \mathrm{~B}_{2}, \ldots$ of pitch classes. These sets are related with each other by symmetries from $T / I$ and thereby define a network of pitch class sets, instead of single pitch classes.

Before leaving this "experimental" context created by Klumpenhouwer and Lewin, we should state a minor result concerning the size of all networks which pertain to a fixed diagram, i.e, all strongly isographic networks.

Proposition 1 Let $\mathcal{D}$ be a diagram with connected diagram scheme $\Delta$. Then the set $\lim \mathcal{D}$ of networks of $\mathcal{D}$ is either empty or is in bijection with a subgroup of $\mathbb{Z}_{12}$. In particular, its cardinality is either 0 or a divisor of 12 .

Proof. Suppose that $\lim \mathcal{D}$ is not empty. Then if $v_{0}$ is a fixed vertex, the projection $p_{v_{0}}: \lim \mathcal{D} \rightarrow \mathcal{D}\left(v_{0}\right)=\mathbb{Z}_{12}$ is an injection, since the diagram scheme is connected and all transition operations $\mathcal{D}(a): \mathcal{D}(x) \rightarrow \mathcal{D}(y)$ are bijections from the group $T / I$. Now, if $t$., $s$. are two networks, their difference $t$. $-s$. is a network for the diagram $\mathcal{D}^{0}$, which is derived from $\mathcal{D}$ by taking for every arrow $a$ the linear part $p= \pm 1$ of $\mathcal{D}(a)=e^{t} p$. Conversely, for every network $d . \in \lim \mathcal{D}^{0}$, the $\operatorname{sum} s .+d$. is a network of $\mathcal{D}$. But the set $\lim \mathcal{D}^{0}$ of networks of the linear diagram is a group under componentwise addition, and its projection $p_{v_{0}}: \lim \mathcal{D}^{0} \rightarrow \mathcal{D}\left(v_{0}\right)=\mathbb{Z}_{12}$ is an injective group homomorphism, so $\lim \mathcal{D}^{0}$ is isomorphic to a subgroup of $\mathbb{Z}_{12}$, as claimed.

The following example in figure 6 shows that for non-commutative dia- 
grams, the number of strongly isographic K-nets is a proper divisor (4) of 12 , and not 12 as in the case of a commutative diagram.


Figure 6: The four solutions (strongly isographic K-nets) of this diagram illustrate that the cardinality of the solution set is a divisor of 12 . Here, the operator $M_{7}$ denotes the multiplication by 7 .

\section{Forms and Denotators for Networks}

The point of the network approach is that due to its underlying universality in the sense of category theory, there is absolutely no reason to stick to the space $\mathbb{Z}_{12}$ of pitch classes, nor is it necessary to have such limited algebraic structures as finite cyclic groups. We now propose a generalization which not only enables more flexible applications, but which will also imply the construction principle for recursively defined networks. This will be discussed in section 5 .

The first step in generalizing Klumpenhouwer-Lewin networks concerns the target category $T / I$. In our tradition of applying modern mathematics to music theory (often coined MaMuTh), one usually considers more general supporting spaces than merely $\mathbb{Z}_{12}$. For example, one takes a fourdimensional real vector space $M=\mathbb{R}^{4}$, where the coordinates represent onset $o$, pitch $p$, loudness $l$, and duration $d$, in an appropriate parametrization by real numbers $o, p, l, d \in \mathbb{R}$. The common basis of such structures is provided by modules $M, N, \ldots$ over a fixed commutative ring $R$ with unity 1 , together with affine maps $f: M \rightarrow N$, which are by definition of the form $f=e^{n} g$, where $n \in N$ and $g: M \rightarrow N$ is $R$-linear. ${ }^{5}$ This means that for

\footnotetext{
${ }^{5}$ An $R$-module $M$ is by definition an additive commutative group $M$, together with a scalar multiplication $R \times M \rightarrow M:(r, m) \mapsto r m$ such that $(r+s) m=r m+s m$ and $r(m+n)=r m+r n$ and $1 m=m$, for all $r, s \in R, m, n \in M$. This generalizes the wellknown structure of a vector space if the coefficients are elements of a general commutative ring $R$ instead of a field.
} 
$x \in M$, we have $f(x)=n+g(x)$. Affine maps may be composed just as set maps are, and we obtain the category $\operatorname{Mod}_{R}$ of $R$-modules with affine maps. In this context, a diagram is a map $\mathcal{D}: \Delta \rightarrow \mathbf{M o d}_{R}$ associating with every vertex $v \in V$ of the diagram scheme $\Delta$ a $R$-module $\mathcal{D}(v)$, and with every arrow $x \stackrel{a}{\longrightarrow} y$ of $\Delta$ an affine map $\mathcal{D}(a): \mathcal{D}(x) \rightarrow \mathcal{D}(y)$. Commutativity conditions are defined in the same way as above. We may now again define a network for the diagram $\mathcal{D}$ as an element of $\lim \mathcal{D}$. Again, we have this type of description of the networks for $\mathcal{D}$ :

Proposition 2 Let $\mathcal{D}$ be a diagram in the category of $R$-modules with diagram scheme $\Delta$ and vertex set $V$. Then the set $\lim \mathcal{D}$ of networks of $\mathcal{D}$ is either empty or is in bijection with a $R$-submodule of the direct product module $\prod_{v \in V} \mathcal{D}(v)$. If $\Delta$ is connected and all transition morphisms are isomorphisms, then $\lim \mathcal{D}$ is either empty or in bijection with a submodule of any of its vertex modules $\mathcal{D}(v)$.

Although this setup looks quite general, it has serious limitations which impose themselves if one tries to build networks of networks of networks etc. In fact, $R$-module networks of a given diagram do not, in general, define a new $R$-module. Moreover, if we step over to the powerset diagrams $2^{\mathcal{D}}$, we loose all the algebraic (module) structure we were given for the single element networks. Thirdly, more complex objects, such as dodecaphonic series or similar parametrized collections of pitch classes, say, cannot be dealt with in this setup. The failure is not by case, since all the constructions related to powersets must fail for categories of modules. The deeper reason for this is that categories of modules $\operatorname{Mod}_{R}$ are not topoi. We shall not open this farout field of modern mathematics here, but just want to stress the limitation of classical approaches when creating objects which should be tractable by typical universal constructions, such as power objects (generalizing function sets $X^{Y}$ from set theory), or limits.

This mathematically motivated limitation is one of the main reasons why we have developed (and implemented in the musical analysis and performance software RUBATO ${ }^{\circledR}[18]$ ) the formalism of denotators. ${ }^{6}$ We shall not develop here the full formalism of denotators, but only highlight the category-theoretic aspect of building new spaces upon given spaces by means of limit and power object constructions. In particular, we shall not deal with name spaces, and we shall also omit completely the colimit construction in denotator theory. However, one should retain that named spaces are what

\footnotetext{
${ }^{6}$ See the paper "The Topos Geometry of Musical Logic" in [2] for a short presentation of the denotator formalism in relation with a "Galois theory of concepts".
} 
in [19] are called forms, while denotators are essentially named elements of such forms. The defective situation which on one side produces non-algebraic structures by powersets and on the other does not allow for more complex parametrized objects is overcome by the standard Yoneda construction of presheaves as we show in the following section.

\section{Yoneda Philosophy in Presheaf Categories}

Suppose that for a fixed commutative ring $R$, we are given the category $\operatorname{Mod}_{R}$ of $R$-modules and affine maps. A presheaf over $\operatorname{Mod}_{R}$ is a contravariant functor $F: \operatorname{Mod}_{R} \rightarrow$ Sets with values in the category Sets of sets. This means that the following conditions hold:

- for every module $M$, also called an address, we are given a set $F(M)$, whose elements are called $M$-addressed points of $F$.

- For every affine map $f: M \rightarrow N$, we are given a set map (attention: arrow directions are reversed!) $F(f): F(N) \rightarrow F(M)$.

- If $f=I d_{M}$ is the identity on $M$, then $F\left(I d_{M}\right)=I d_{F(M)}$ is the identity on the image set $F(M)$.

- If $f: M \rightarrow N, g: N \rightarrow L$ are two affine maps, then we have $F(g \circ f)=$ $F(f) \circ F(g)$ (attention, by reversed arrows, composition of images also reverses order!).

The most important examples of presheaves are those defined by modules. Consider for a fixed $R$-module $X$, and for a module $M$ the set of affine maps $f: M \rightarrow X$. We use the notation $M @ X$ for this set in order to make evident that we are looking "at $X$ " from the "perspective" of the module $M$. This assignment defines a presheaf $@ X: \operatorname{Mod}_{R} \rightarrow$ Sets. If $f: M \rightarrow N$ is an affine map, then $f @ X: N @ X \rightarrow M @ X$ maps $g: N \rightarrow X$ to the composition $f @ X(g)=g \circ f: M \rightarrow X$. It is well known that the system of presheaves defines a category $\operatorname{Mod}_{R}^{@}$ whose morphisms are the natural transformations among presheaves, see [6].

Then we have the famous Yoneda embedding $Y: \operatorname{Mod}_{R} \rightarrow \operatorname{Mod}_{R}^{@}$, which sends a module $X$ to its presheaf $@ X$. This embedding has the remarkable property that it reflects isomorphisms, i.e., two modules $X, Y$ are isomorphic if and only if their presheaves @ $X, @ Y$ are so. In other words, we do not loose any information while working in $\mathbf{M o d}_{R}^{@}$ instead of $\mathbf{M o d}_{R}$. More precisely, if $f: X \rightarrow Y$ is an affine map, then the associated natural 
transformation @ $f: @ \rightarrow @ Y$ maps an affine map $g: M \rightarrow X$ to the map $@ f(g)=f \circ g$. The assignment $f \mapsto @ f$ then defines a bijection $X @ Y \stackrel{\sim}{\rightarrow}$ Nat(@X,@Y), where Nat $(@ X, @ Y)$ is the set of natural transformations from the presheaf $@ X$ to the presheaf $@ Y$.

This fact is particularly evident for the reconstruction of $f$ from $@ f$. Here is the procedure. To begin with, the evaluation of @X at the zero $R$-module 0 yields $@ X(0)=0 @ X=\left\{e^{x} 0 \mid x \in X\right\}$ i.e., we have a bijection $X \stackrel{\sim}{\rightarrow} 0 @ X: x \mapsto e^{x} 0$. Then, if $f: X \rightarrow Y$ is an affine map, we have $0 @ f\left(e^{x} 0\right)=f \circ e^{x} 0=e^{f(x)} 0$. This means that the bijections $X \stackrel{\sim}{\rightarrow} 0 @ X, Y \stackrel{\sim}{\rightarrow}$ $0 @ Y$ induce exactly $f: X \stackrel{\sim}{\rightarrow} Y$, and we have recovered $f$ from the evaluation of the presheaves@X,@Y at the zeromodule.

The presheaf category has all nice properties we need to build limits, colimits, power objects, and a subobject classifier; it is in fact a topossee. ${ }^{7}$ More concretely, the limits and colimits of a diagram $\mathcal{D}: \Delta \rightarrow \operatorname{Mod}_{R}^{@}$ are calculated pointwise, i.e., for every module $M$, we have $(\lim \mathcal{D})(M)=\lim (\mathcal{D}(M))$, the limit of the corresponding diagram of sets, which results from the evaluation of the diagram's presheaves and the connecting natural transformations. The same holds for colimits. Only the powerset presheaf is a bit more involved. If $F$ is any presheaf, then its powerset is the presheaf $\Omega^{F}$, whose value for a module $M$ is $\Omega^{F}(M)=\{G \mid G \subset @ M \times F$ is a subpresheaf $\}$. There are two subpresheaves of $\Omega^{F}$, which are frequently used, i.e., the subset presheaf $2^{F}$, where $2^{F}(M)=2^{F(M)}$, and its subpresheaf of finite subsets, i.e., $\operatorname{Fin}(F) \subset 2^{F}$ with $\operatorname{Fin}(F)(M)=\{X \mid X \subset F(M), \operatorname{card}(X)<\infty\}$. Although $2^{F}$ is a natural construction, it does not have the characteristic property of a powerset object, but see [19] for details. In the sequel, we shall assume that, given a presheaf $F, P(F)$ is typically one of the three powerset constructions $\Omega^{F}, 2^{F}$, or $\operatorname{Fin}(F)$, but other natural subpresheaves may intervene, see [20].

We now show how these tools enable us to extend the concept of Klumpenhouwer networks in the context of presheaf categories. Reconsider the original setup, where we had a diagram $\mathcal{D}: \Delta \rightarrow T / I$. The target category $T / I$ is a subcategory of the category $\mathbf{M o d}_{\mathbb{Z}}$ of $\mathbb{Z}$-modules (recall that

\footnotetext{
${ }^{7}$ Topoi were introduced by Alexander Grothendieck in the context of modern algebraic geometry, then applied to questions of logic and the foundation of mathematics by William Lawvere, see [7]. A topos is a category with finite limits, colimits, power objects, and a subobject classifier. This generalizes well-known properties from (mathematical) set theory and thereby unifies geometric and logical concepts and techniques. An important topos in our application to networks is the topos of directed graphs. This fact implies that networks may be interpreted as logical constructions within a topos-theoretical universe, whose musical consequences must be investigated by future research.
} 
these are just additively written abelian groups). So let us work in this latter category. According to the previous Yoneda formalism, we may embed $\operatorname{Mod}_{\mathbb{Z}}$ in the presheaf category $\mathbf{M o d}_{\mathbb{Z}}^{@}$. Restating the diagram $\mathcal{D}$ in terms of presheaves, we get a new diagram $\mathcal{D}^{@}: \Delta \rightarrow \operatorname{Mod}_{\mathbb{Z}}^{@}$ by a map $\mathcal{D}^{@}(v)=@(\mathcal{D}(v))=@ \mathbb{Z}_{12}$, whereas for an arrow $x \stackrel{a}{\longrightarrow} y$, we have the transition operations $\mathcal{D}^{@}(a)=@(\mathcal{D}(a))$. Therefore, according to the previous constructions, if $M$ is a $\mathbb{Z}$-module, and if $f: M \rightarrow \mathbb{Z}_{12}$ is an element of $M @ \mathbb{Z}_{12}$, then $\mathcal{D}^{@}(a)(f)=\mathcal{D}(a) \circ f$.

Let us now consider the limit of this presheaf diagram $\mathcal{D}^{@}$. We have already seen that the evaluation of the limit at a module $M$ is the limit of the diagram's evaluation at $M$. Therefore, if $V$ denotes as before the vertex set of the diagram scheme $D$, an element of $\lim \mathcal{D}^{@}(M)$ is a family $t .=\left(t_{v}\right)_{v \in V}$, where

- for all $v \in V, t_{v}: M \rightarrow \mathbb{Z}_{12}$ is an affine map, i.e., $t_{v}=e^{s_{v}} p_{v}$, with $s_{v} \in \mathbb{Z}_{12}$ and $p_{v}: M \rightarrow \mathbb{Z}_{12}$ a homomorphism of abelian groups;

- for every arrow $x \stackrel{a}{\longrightarrow} y$, we have $t_{y}=\mathcal{D}(a) \circ t_{x}$, where $\mathcal{D}(a) \in T / I$.

We call an element $t . \in \lim \mathcal{D}^{@}(M)$ a $M$-addressed network of $\mathcal{D}^{@}$, whereas the modules $M$ are called the address of this network. Clearly, 0 -addressed networks are precisely the classical Klumpenhouwer-Lewin networks. But the more general situation is new and opens a wide spectrum of generalized networks. This functorial point of view was introduced in [19] because the restriction to the zero address cannot cope with a number of genuinely music(ologic)al problems.

A simple, but typical, example is the conceptualization of dodecaphonic series. A dodecaphonic series may be defined as a sequence $S=\left(s_{0}, s_{1}, \ldots s_{11}\right)$ of pairwise different pitch classes $s_{i} \in \mathbb{Z}_{12}$, where the order position index $i$ is something like an abstract timeline, see [19, chapter 8.1.1, definition 22]. But we have a bijection $\mathbb{Z}^{k} @ M \stackrel{\sim}{\rightarrow} M^{k+1}: f \mapsto\left(f\left(e_{0}\right), f\left(e_{1}\right), f\left(e_{2}\right), \ldots f\left(e_{k}\right)\right)$, where $e_{0}=0, e_{1}=(1,0, \ldots 0), e_{2}=(0,1,0, \ldots 0), e_{k}=(0,0, \ldots 0,1)$. So a series $S$ is identified with the affine map $S: \mathbb{Z}^{11} \rightarrow \mathbb{Z}_{12}$ with $S\left(e_{i}\right)=$ $s_{i}, i=0,1, \ldots 11$, and this is just a $\mathbb{Z}^{11}$-addressed point of $@ \mathbb{Z}_{12}$. Then a $\mathbb{Z}^{11}$-addressed network of $\mathcal{D}$ is a network, whose vertexes are $\mathbb{Z}^{11}$-addressed points of $@ \mathbb{Z}_{12}$, and if all these vertexes are dodecaphonic series $\left(s_{i} \neq s_{j}\right.$ for $i \neq j$ ), it is a network of dodecaphonic series. These series are connected by operations from the group $T / I$, which establishes a well-kown network of series in the classical sense of dodecaphonism. But there is more! In fact, the functoriality of a presheaf has not been used explicitely. We subsume this aspect under the operation of "address change" in presheaves, which 
means that we consider affine maps $\alpha: M \rightarrow N$ of modules, and the associated maps $F(\alpha): F(N) \rightarrow F(M)$. In our case, we may in particular consider the affine map $\rho: \mathbb{Z}^{11} \rightarrow \mathbb{Z}^{11}$, which is defined by $\rho\left(e_{i}\right)=e_{11-i}$ for all $i=0,1, \ldots 11$. This is the retrograde map, since it reverses time indexes. (It is easily seen that any permutation of the basis elements $e_{i}$ defines an affine map on the address $\mathbb{Z}^{11}$. Therefore any rearrangement of the time indexes is induced by an address change.) Now, if $S: \mathbb{Z}^{11} \rightarrow \mathbb{Z}_{12}$ is any series, the retrograde series $\rho(S)=S \circ \rho$ is deduced from $S$ by the address change $\rho: \mathbb{Z}^{11} @ \mathbb{Z}_{12} \rightarrow \mathbb{Z}^{11} @ \mathbb{Z}_{12}$. This implies that by an address change $\rho$, we obtain the retrograde series network $\rho\left(S\right.$.) of a network $S .=\left(S_{v}\right)_{v \in V}$ of dodecaphonic series $S_{v}$. Let us terminate this address change topic by the example $\epsilon_{i}=e^{e_{i}} 0: 0 \rightarrow \mathbb{Z}^{11}$, sending 0 to the $i$-th basic element $e_{i}, i=0,1, \ldots 11$. If we apply this base change to the network $S$., we obtain the 0 -addressed network $\epsilon_{i}(S$.), which is a classical network and consists of the $i$-th entries of the series, from which $S$. is built. The conclusion we can draw form the previous example can be stated in the form of the following economy principle:

The functorial setup prevents us from reinventing the concept of a network for each new address, i.e., we are given a whole bunch of variants of addressed networks. Moreover, the networks of different addresses may be functorially related by the address changes.

\section{The General Recursion Principle}

The previous discussion has shown the first advantages of the categorical restatement and generalization of the network idea. We are however not ready to address the recursion problem: What should be a network of networks of networks, and so on? Do we have to invent a new conceptual environment each time we iterate the networking process? We do not, and this is the main statement of this final section.

Let us recapitulate the status quo. We have seen that one may start from a diagram $\mathcal{D}: \Delta \rightarrow \operatorname{Mod}_{R}^{@}$ of presheaves over a module category $\operatorname{Mod}_{R}$, and then consider the $\operatorname{limit} \lim \mathcal{D}$ and its $M$-addressed points as networks. Observe that in the case of values in representable ${ }^{8}$ presheaves $\mathcal{D}(v)=@ M_{v}$ on vertexes $v \in V$, such as we have dealt with for the pitch class module $M_{v}=\mathbb{Z}_{12}$ in the classical example from the KlumpenhouwerLewin theory, we have not taken into account networks of pitch class sets.

\footnotetext{
${ }^{8}$ By definition, a representable presheaf is one which is isomorphic to a presheaf of the form @ $M$ for a module $M$.
} 
This can be achieved if we do not directly take the limit of $\mathcal{D}$, but the limit of one of the above powerset presheaf construction $P$. This means that we take the powerset diagram $P(\mathcal{D})$ in the following sense: If $v$ is a vertex in $\Delta$, we define $P(\mathcal{D})(v)=P(\mathcal{D}(v))$, and if $x \stackrel{a}{\longrightarrow} y$ is an arrow of $\Delta$, we set $P(\mathcal{D})(a)=P(\mathcal{D}(a))$. For example, if $P(F)=2^{F}$, then $2^{\mathcal{D}}(a)=2^{\mathcal{D}(a)}$, the map which takes a subset $X \subset \mathcal{D}(x)$ to the subset $\mathcal{D}(a)(X) \subset \mathcal{D}(y)$. So far, we have left the case, where diagram vertexes are representable by modules, i.e., $\mathcal{D}(v)=@ M_{v}$. But we are still in the context of a diagram with values in the category of presheaves over $\operatorname{Mod}_{R}$, namely $P(\mathcal{D}): \Delta \rightarrow \operatorname{Mod}_{R}^{@}$. Let us work with this powerset diagram and its $\operatorname{limit} \lim P(\mathcal{D})$. A network of networks is defined as follows. We consider two diagrams $\mathcal{D}_{1}, \mathcal{D}_{2}: \Delta \rightarrow$ $\operatorname{Mod}_{R}^{@}$ and then a natural transformation $g: \mathcal{D}_{1} \rightarrow \mathcal{D}_{2}$ of diagrams. Recall that this means defining a natural transformation $g(v): \mathcal{D}_{1}(v) \rightarrow \mathcal{D}_{2}(v)$ for each vertex $v$ such that the diagram of presheaves

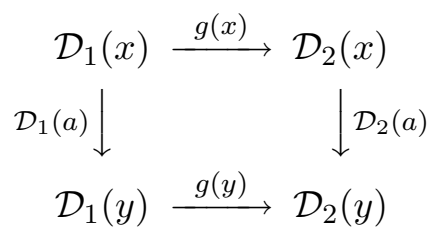

commutes for each arrow $x \stackrel{a}{\longrightarrow} y$. This one induces a morphism

$$
\lim g: \lim \mathcal{D}_{1} \rightarrow \lim \mathcal{D}_{2}
$$

of the limit presheaves. This means in particular that we have a set map of $M$-addressed networks

$$
\lim g(M): \lim \mathcal{D}_{1}(M) \rightarrow \lim \mathcal{D}_{2}(M)
$$

for every address $M$, and under such a map, $M$-addressed networks for $\mathcal{D}_{1}$ are related to $M$-addressed networks for $\mathcal{D}_{2}$. But basically, the context is not different from the initial one! We still have presheaves (the limits) and we still have natural transformations between such presheaves (the limits of diagram morhisms). This induces the following construction process:

1. Fix a diagram scheme $\Delta$ and one type $P$ of powerset construction for presheaves.

2. Suppose that we are given a category $\mathcal{C} \subset \mathbf{M o d}_{R}^{@}$ of presheaves (not necessarily all presheaves, not necessarily all natural transformations between these presheaves, but containing the identities and closed under composition). 
3. Take the category $\operatorname{Diagr}(\Delta, \mathcal{C})$ of diagrams $\mathcal{D}: \Delta \rightarrow \mathcal{C}$.

4. Take the $\operatorname{limits} \lim P(\mathcal{D})$ of these diagrams as objects of a new network category $\Delta \bullet_{P} \mathcal{C}$, together with the limits of the natural transformations (over $\mathcal{C}$ ) between powersets of such diagrams in $\operatorname{Diagr}(\Delta, \mathcal{C})$ as morphisms.

This process is the core operation in the definition of iterated networks. In fact, the resulting category $\mathcal{C}^{1}=\Delta \bullet_{P} \mathcal{C}$ of the above operation is by no means different in quality from the initial category $\mathcal{C}$, it is just another subcategory of $\operatorname{Mod}_{R}^{@}$. So we may go on and select a new diagram scheme $\Delta^{1}$, a new powerset construction $P^{1}$, and the network category $\mathcal{C}^{1}$. We then obtain the second network category $\mathcal{C}^{2}=\Delta^{1} \bullet_{P^{1}} \mathcal{C}^{1}$, and so on. This construction can be repeated without any change and yields networks of networks of networks, ad infinitum. Let us add that this type of iterated construction of spaces by means of limits, but more generally also by means of colimits, and powersets, is the basic tool in the systematic concept framework of denotators and forms in [19].

We are left with the following fundamental, still unsettled, problem: Suppose that one has the iterated framework category $\mathcal{C}^{2}=\Delta^{1} \bullet_{P} \mathcal{C}^{1}$, using the same powerset construction $P^{1}=P$ as for $\mathcal{C}^{1}=\Delta \bullet_{P} \mathcal{C}$. Is it always possible to find a diagram scheme $\Delta^{1} \diamond_{P} \Delta$, built from the given diagram schemes $\Delta^{1}$ and $\Delta$, and possibly depending on $P$, such that we have an isomorphism

$$
\left(\Delta^{1} \diamond_{P} \Delta\right) \bullet_{P} \mathcal{C} \stackrel{\sim}{\rightarrow} \Delta^{1} \bullet_{P}\left(\Delta \bullet \bullet_{P} \mathcal{C}\right)
$$

of framework categories? If this is true, one may replace iterated framework constructions by one-step frameworks by use of a directed graph construction, which is much easier than iterated categories. The other way round: Which are the (types of) directed graphs where iterated networks can be realized by a $\Delta^{1} \diamond_{P} \Delta$ construction?

\section{Some Remarks on Categories of Networks}

The previous exposition suggests that one should introduce and study categories of networks. Their objects would be networks (possibly of variable addresses) and built upon variable diagram schemes. Morphisms would then be induced by morphisms of diagram schemes, coupled with natural transformations between adequate diagrams. This idea is developed in [20]. 
A second remark concerns the dichotomy of local and global objects, which are known to describe structures in MaMuTh. The local-global dichotomy can also be realized for the level of "processes" described by networks, instead of the level of "structures" as described by local/global compositions. This means that we now may glue "local" networks to "global" neworks. This approach has also been introduced in [20].

The future research must of course deal with the relations between structures and processes. More precisely, this question concerns the existence of functors from the categories of local/global networks to the categories of local/global compositions. In [20], it is shown that there is such a functor, which associates with every global network an underlying global composition. This result implies that there are proper global networks which cannot be reduced to local networks by the well-known interpretational process. Such a process is realized by the covering of a given local object by an atlas of local charts. This functor yields a number of invariants for the classification of global networks, which means that the classification of global networks may use the classification of global composition described in [19, chapter $15]$.

Although the coincidence of Klumpenhouwer networks with elements of categorical limits invokes classical instances of category theory, it is remarkable that music theory has "rediscovered" the idea of such basic structures! There is however a lesson to be drawn from this amusing fact: Music theory would draw a great profit from the systematic subsidiary study of modern mathematics. 


\section{References}

[1] Andreatta, Moreno: Méthodes algébriques en musique et musicologie du XXème siècle : aspects théoriques, analytiques et compositionnels. PhD thesis, Ircam/EHESS 2003

[2] Assayag, Gérard et al. (eds.): Mathematics and Music. Springer, Heidelberg et al. 2002

[3] Bent, Ian: Article "Analysis" in: The New Grove, MacMillan, London 1980

[4] Foley, Gretchen: Arrays and K-nets: Transformational relationships within Perles Twelve-Tone Tonality. Music Theory Midwest, May 2003

[5] Headlam, Dave: Perle's Cyclic Sets and Klumpenhouwer Networks: A Response. Music Theory Spectrum 24.2: 246-56, 2002

[6] Mac Lane, Saunders: Categories for the Working Mathematician. Springer, Heidelberg et al. 1971

[7] Mac Lane, Saunders and Ieke Moerdijk: Sheaves in Geometry and Logic. Springer, New York et al. 1994

[8] Klumpenhouwer, Henry: Deep Structure in K-net Analysis with special reference to Webern's opus 16,4. Unpublished manuscript

[9] Klumpenhouwer, Henry: A Generalized Model of Voice-Leading for Atonal Music. Ph.D. Thesis, Harvard University 1991

[10] Lewin, David: Transformational Techniques in Atonal and other Music Theories. Perspectives of New Music, 21, pp. 312-371, 1982

[11] Lewin, David: Generalized Musical Intervals and Transformations. Yale University Press, New Haven 1987

[12] Lewin, David: Klumpenhouwer Networks and Some Isographies that Involve Them. Music Theory Spectrum 12/1, 1990

[13] Lewin, David: Musical Form and Transformation: 4 Analytic Essays. Yale University Press, New Haven and London 1993

[14] Lewin, David: A Tutorial on Klumpenhouwer Networks, Using the Chorale in Schoenbergs Opus 11, No. 2. Journal of Music Theory 38.1: 79-101, 1994 
[15] Lewin, David: Thoughts on Klumpenhouwer Networks and PerleLansky Cycles. Music Theory Spectrum 24.2: 196-230, 2002

[16] Mazzola, Guerino: Gruppen und Kategorien in der Musik. Heldermann, Berlin 1985

[17] Mazzola, Guerino: Geometrie der Töne. Birkhäuser, Basel et al. 1990

[18] Mazzola, Guerino et al.: RUBATO on the Internet. http://www.rubato.org, Univ. Zürich 1996

[19] Mazzola, Guerino et al.: The Topos of Music - Geometric Logic of Concepts, Theory, and Performance. Birkhäuser, Basel et al. 2002

[20] Mazzola, Guerino: Local and Global Limit Denotators and the Classification of Global Compositions. In: Fripertinger H, Reich L (eds.): Colloquium on Mathematical Music Theory. Grazer Math. Ber., ISSN 1016-7692, Bericht Nr. 347 (2004), 1-9

[21] Mazzola, Guerino et al.: Comprehensive Mathemtatics for Computer Scientists I, II. Springer, Heidelberg et al. 2004

[22] Morris, Robert D.: Class Notes for Advanced Atonal Music Theory. Frog Peak Music, Labanon 2001

[23] Peel, John: On some celebrated measures of the Schoenberg String Trio. Perspectives of New Music, 14/15 : 260-279, 1975

[24] Perle, George: Letter to the editor. Music Theory Spectrum 15.2 30003, 1993

[25] Roeder, John: A MaMuTh Achievement (Review of Guerino Mazzola's Geometrie der Tne and Gruppen und Kategorien in der Musik). Perspectives of New Music 31/2: 294-312, 1993 